\title{
ANÁLISE FITOSSOCIOLÓgICA DA FLORESTA NACIONAL DE PACOTUBA, CACHOEIRO DE ITAPEMIRIM, ES - BRASIL
}

\author{
Karla Maria Pedra de Abreu ${ }^{1}$, Gilson Fernandes da Silva², Aderbal Gomes da Silva ${ }^{3}$
}

(recebido: 7 de outubro de 2010; aceito: 29 de novembro de 2012)

\begin{abstract}
RESUMO: Neste trabalho, objetivou-se avaliar a estrutura da comunidade arbórea da Floresta Nacional de Pacotuba, em Cachoeiro de Itapemirim - ES. Foram alocadas, sistematicamente, 12 parcelas de 40x50m, sendo medidas e identificadas todas as árvores vivas com DAP $\geq 5,0 \mathrm{~cm}$. A análise fitossociológica foi baseada nos parâmetros absolutos e relativos de densidade, dominância, frequência, valor de importância e área basal. A estrutura diamétrica foi analisada a partir de histogramas com intervalos de classes definidos pela fórmula de Spiegel. Foram amostrados 3570 indivíduos, 253 espécies e 52 famílias. As espécies com maior valor de importância (VI) foram Senefeldera verticillata, Actinostemon estrellensis, Anadenanthera peregrina. As curvas (J-reverso) e valores do quociente "q" indicaram ausência de problemas de regeneração. $\mathrm{O}$ valor de área basal $\left(25,72 \mathrm{~m}^{2} . \mathrm{ha}^{-1}\right) \mathrm{e}$ a alta dominância de poucas espécies permitem classificar esse fragmento como em estádio intermediário de sucessão. Entretanto, destaca-se sua expressiva riqueza, a presença de espécies de importância conservacionista e a necessidade dessa área ser priorizada em programas de manejo e conservação na região.
\end{abstract}

Palavras-chave: Fragmentação, Floresta Estacional Semidecidual, fitossociologia.

\section{PHYTOSOCIOLOGICAL ANALYSIS OF PACOTUBA NATIONAL FOREST, CACHOEIRO DE ITAPEMIRIM, ES - BRASIL}

\begin{abstract}
This study evaluated the structure of the tree community of Pacotuba National Forest, in Cachoeiro de Itapemirim - ES. For the phytossociological inventory, 12 sample plots of $40 \times 50$ m each were systematically laid out. All trees with DBH $\geq 5 \mathrm{~cm}$ were measured and identified. The phytossociological analysis was based on the absolute and relative parameters of density, dominance, frequency, importance values and basal area values. The diametric structure was analyzed using diameter class histograms defined by Spiegel's formula A total of 3570 individuals, 253 species and 52 families were sampled. The species with the highest importance value (IV) were: Senefeldera verticillata, Actinostemon estrellensis, Anadenanthera peregrina. The diametric curves (J-reverse shape) and values of Liocourt's ' $q$ ' quotient suggested problems involving lack of regeneration. The value of basal area $\left(25,72 \mathrm{~m}^{2} . h a^{-1}\right)$ and the highest dominance offew species classified this fragment as in the intermediate stage of succession. However, due to the expressive species richness and the presence of some endangered tree, this remnantforest should be considered as a priority area for establishment of conservation and management practices.
\end{abstract}

Key words: Fragmentation, Semideciduous Seasonal Forest, phytosociology.

\section{INTRODUÇÃO}

Em várias regiões tropicais, o processo da fragmentação florestal vem aumentando em função das altas taxas de desmatamento (WHITMORE, 1997). A Mata Atlântica, atualmente, está reduzida a 10,4\% de sua cobertura original (FUNDAÇÃO SOS MATA ATLÂNTICA; INSTITUTO NACIONAL DE PESQUISAS ESPACIAIS - INPE, 2009), sendo um exemplo desse processo, visto que esta ocupava cerca de 150 milhões de hectares no território brasileiro (RIBEIRO et al., 2009). Os impactos das atividades de exploração econômica e da alta densidade demográfica fizeram com que a Mata Atlântica fosse reduzida drasticamente (MORELLATO; HADDAD, 2000).

No estado do Espírito Santo, Schettino e Gonçalves (2002) ressaltam que as madeiras utilizadas para os mais diversos fins foram obtidas dos remanescentes florestais nativos com elevadas taxas de desmatamento, o que tem contribuído para uma exaustão dos recursos florestais nessa região. A Floresta Estacional Semidecidual é a segunda formação vegetacional mais importante em termos de área no Estado do Espírito Santo (INSTITUTO DE PESQUISAS DA MATAATLÂNTICA- IPEMA, 2005). Na região sul do referido estado, essa fitofisionomia está reduzida a remanescentes tipicamente pequenos, isolados

\footnotetext{
${ }^{1}$ Bióloga, Professora Doutoranda em Ecologia e Recursos Naturais - Instituto Federal do Espírito Santo/IFES - Cx. P. 47 - 29520-000 - Alegre, ES, Brasil - kmpaarchanjo@ifes.edu.br

${ }^{2}$ Engenheiro Florestal, Professor Pós-Doutorado em Manejo Florestal - Universidade Federal do Espírito Santo/UFES - Centro de Ciências Agrárias - Departamento de Engenharia Florestal - Cx. P. 16 - 29550-000 - Jerônimo Monteiro, ES, Brasil - gfsilva@yahoo.com ${ }^{3}$ Engenheiro Florestal, Professor Doutor em Ciências Florestais - Universidade Federal do Espírito Santo/UFES - Centro de Ciências Agrárias - Departamento de Engenharia Florestal - Cx. P. 16 - 29550-000 - Jerônimo Monteiro, ES, Brasil - aderbalsilva@yahoo.com.br
} 
e alterados, formados a partir da ocupação pela cultura do café, cana de açúcar, prática da pecuária, exploração e beneficiamento de rochas ornamentais. Nascimento et al. (2006) destacam que a atividade desordenada de ocupação da terra foi um fator motivador para o processo de devastação da paisagem natural do extremo sul capixaba. A fragmentação florestal pode ser prejudicial para as comunidades vegetais remanescentes, não apenas pela redução de sua área original, mas também pelos seus efeitos (CARVALHO et al., 2009), tais como: efeito de borda, impedimento ou redução na taxa de migração, diminuição do tamanho populacional com perda de variabilidade genética (CORDEIRO; HOWE, 2001; FAHRIG, 2003; LAURANCE et al., 2002, 2006; MELO et al., 2006; METZGER et al., 2009; MURCIA, 1995).

Visto que os fragmentos florestais são mais susceptíveis às perturbações antrópicas e alterações bióticas, que podem se refletir na composição e estrutura da comunidade arbórea, os mesmos merecem atenção especial (LAURANCE; YENSEN, 1991; WHITMORE; BURSLEM, 1998). Vários autores têm ressaltado a necessidade de se estudar as comunidades vegetais a fim de se obter um norteamento quanto às decisões para a conservação e manejo dessas áreas (CARVALHO et al., 2006, 2007; DAN et al., 2010; LIBANO; FELFILI, 2006; MARANGON et al., 2007; SOUZA et al., 2012).

As investigações sobre os efeitos da fragmentação têm sido tema de vários estudos atualmente, nos quais a avaliação da riqueza, diversidade, estrutura, grupos ecológicos, ocorrência de espécies raras e ameaçadas são utilizadas para caracterizar o estágio de conservação das áreas inventariadas (CARVALHO et al., 2009; DAN et al., 2010; PESSOA; OLIVEIRA, 2006; PINTO et al., 2007; SOUZA et al., 2012).

Verifica-se uma lacuna de conhecimento sobre a vegetação arbórea da região sul do Espírito Santo (OLIVEIRA-FILHO; FONTES, 2000) que, apesar de apresentar um quadro de fragmentação intenso, ainda possui áreas naturais relevantes que merecem esforços de proteção. Diante do exposto, objetivou-se, no presente trabalho, analisar a estrutura fitossociológica da comunidade arbórea de um fragmento florestal de Floresta Estacional Semidecidual no sul do Espírito Santo, a Floresta Nacional de Pacotuba.

\section{MATERIAL E MÉTODOS}

\section{1 Área de estudo}

O presente trabalho foi desenvolvido em um fragmento florestal de 119 hectares da Floresta
Nacional (FLONA) de Pacotuba (20 $45^{\prime}$ S e $41^{\circ} 17^{\prime} \mathrm{W}$ ), a qual possui uma área total de 450 hectares e está situada no município de Cachoeiro de Itapemirim, sul do Espírito Santo (Figura 1).
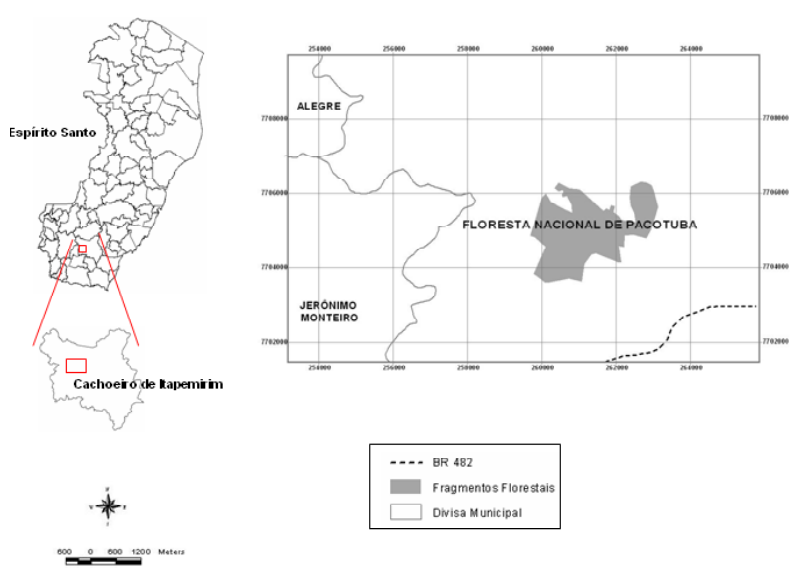

Figura 1 - Ilustração da localização da Floresta Nacional de Pacotuba, Cachoeiro de Itapemirim, Espírito Santo.

Figure 1 - Illustration of Pacotuba National Forest location.

O clima da região é do tipo Cwa (KÖPPEN, 1948), com precipitação média anual de $1293 \mathrm{~mm}$ (INSTITUTO CAPIXABA DE PESQUISA, ASSISTÊNCIATÉCNICAE EXTENSÃO RURAL - INCAPER, 2012). A temperatura média mínima do mês mais frio varia entre 11,8 e $18^{\circ} \mathrm{C}$ e a média máxima do mês mais quente varia entre 30,7 e $34^{\circ} \mathrm{C}$ (PEZZOPANE et al., 2004). O tipo de solo predominante é Latossolo Vermelho-Amarelo Distrófico (LVAd), de acordo com o sistema brasileiro de classificação de solos (EMPRESA BRASILEIRA DE PESQUISA AGROPECUÁRIA- EMBRAPA, 2006).

A vegetação da área estudada é classificada como Floresta Estacional Semidecidual Submontana (VELOSO et al., 1991). Quanto ao uso da terra, predominam pastagens, plantios de café e cana de açúcar, com remanescentes florestais nativos localizados principalmente nos topos dos morros (PROJETO CORREDORES ECOLÓGICOS, 2005). A FLONA de Pacotuba é um dos maiores remanescentes florestais da Bacia do Rio Itapemirim, foi criada pelo Dec $s / n^{\circ}$ de 13 de dezembro de 2002 e, ao longo de sua história, foi alvo de vários impactos de origem antrópica, tais como: caça, cultura de café nas bordas e clareiras, extração de palmito, de lenha e de madeiras nobres. 


\subsection{Obtenção dos dados}

Empregou-se o método de amostragem de parcelas de área fixa, sendo as mesmas distribuídas de forma sistemática na área de estudo (MUELLER-DOMBOIS; ELLENBERG, 1974). Foram alocadas 12 parcelas de 40 x 50 $\mathrm{m}$, obtendo-se uma área amostral de 2,4 hectares. Foram amostrados todos os indivíduos arbóreos com diâmetro igual ou superior a $5 \mathrm{~cm}$. Nos casos de indivíduos perfilhados, as ramificações também foram medidas, desde que estivessem vivas e enquadradas nos critérios de inclusão.

\subsection{Análise dos dados}

Foi utilizado o sistema de classificação da Angiosperm Phylogeny Group - APG (2009) para a circunscrição das espécies nas respectivas famílias botânicas. A identificação das espécies foi realizada por meio da comparação dos espécimes coletados com o acervo do Herbário RB, do Instituto de Pesquisas do Jardim Botânico do Rio de Janeiro, mediante consulta a especialistas e/ou a bibliografia especializada. Foram calculados os índices de Shannon-Weaver (H') e Pielou (J) (KENT; COKER, 1992). A suficiência amostral foi avaliada a partir de curvas de rarefação, obtidas utilizando o programa Ecosim 7 (GOTELLI; ENTSMINGER, 2011).

A análise fitossociológica foi baseada nos parâmetros absolutos e relativos de densidade, dominância, frequência, valor de importância e área basal (MUELLER-DOMBOIS; ELLENBERG, 1974), obtidos por meio do programa Mata Nativa 2.06 (CIENTEC, 2006). Com base em observações de campo e revisão de literatura, as espécies foram classificadas em grupos ecológicos, baseado em Gandolfi et al. (1995), como: Pioneiras (Pi), Secundárias iniciais ( $\mathrm{Si}$ ), Secundárias tardias (St) e sem classificação por carência de informações (NC).

Para a análise da estrutura diamétrica foi utilizado um histograma de frequência, com intervalo de classe determinado a partir da fórmula de Spiegel. A referida fórmula é caracterizada pela sexpressão: $\mathrm{IC}=\mathrm{A} / \mathrm{nc}$, em que: A é amplitude e nc é o número de classes, sendo nc $=1+3,3 \log (\mathrm{n})$, em que $\mathrm{n}$ é número de indivíduos. Para avaliar o recrutamento foi calculado o coeficiente "q" de Liocourt, que consiste na divisão do número de indivíduos em uma determinada classe de diâmetro pelo número de indivíduos em uma classe anterior (FELFILI; REZENDE, 2003). Para a obtenção da tabela de distribuição diamétrica, foi utilizado o programa Mata Nativa 2 (CIENTEC, 2006).

\section{RESULTADOS E DISCUSSÃO}

No levantamento fitossociológico realizado na FLONA de Pacotuba, foram amostrados 3570 indivíduos distribuídos em 253 espécies e 52 famílias botânicas, que produziram uma área basal total de $61,73 \mathrm{~m}^{2} /$ ha (Tabela 1 ).

As curvas de rarefação indicaram que o esforço amostral foi suficiente para caracterizar a riqueza e diversidade florística da vegetação do remanescente estudado (Figura 2).

As famílias mais representativas foram Fabaceae (44 espécies), Sapotaceae (16), Myrtaceae (13), Euphorbiaceae (13), Meliaceae (13). Este padrão corrobora com o descrito para as Florestas Estacionais Semideciduais (FES) da Mata Atlântica (OLIVEIRA-FILHO; FONTES, 2000), especialmente quanto à elevada riqueza das famílias Myrtaceae e Fabaceae, que se destacaram em vários estudos realizados no sudeste brasileiro (CARVALHO et al., 2006; DAN et al., 2010; NASCIMENTO; LIMA, 2008; ROLIM et al., 2006). Os valores de área basal e densidade de indivíduos ficaram próximos dos valores encontrados na maioria dos trabalhos realizados em formações vegetais de tipologia florestal semelhante à estudada (Tabela 2). Entretanto, segundo Conselho Nacional do Meio Ambiente - CONAMA (1994) essa área pode ser considerada como em estágio intermediário de sucessão (entre 10 e $28 \mathrm{~m}^{2}$.ha-1). Os índices de diversidade $(3,31)$ e equabilidade $(0,60)$ foram semelhantes aos de outros estudos realizados em FES consideradas como perturbadas (Tabela 2). De fato, uma grande variação pode ocorrer quando se comparam valores do índice de diversidade em diferentes estudos, mesmo os realizados dentro de uma mesma região fitogeográfica (MARANGON et al., 2007). Essas comparações exigem cautela, visto que, fatores relacionados à sucessão, aos diferentes métodos de amostragem, critérios de inclusão, esforço taxonômico e área total amostrada podem influenciar nos valores desses índices (MARANGON et al., 2007; SILVA et al., 2000). Entretanto, na RPPN Cafundó, considerada como uma área bem conservada localizada a aproximadamente 1,5 $\mathrm{km}$ da área estudada, o valor de diversidade alcançou um valor maior, utilizando-se métodos e área de amostragem semelhantes.

$\mathrm{Na}$ área estudada, o valor de H' obtido $(3,31)$ provavelmente se deve à concentração relativamente elevada de abundâncias em um pequeno número de espécies que dominam a comunidade arbórea. Doze espécies

Cerne, Lavras, v. 19, n. 1, p. 157-168, jan./mar. 2013 
Tabela 1 - Parâmetros fitossociológicos e categorias sucessionais das espécies arbóreas amostradas na FLONA de Pacotuba, Cachoeiro de Itapemirim, ES.

Table 1 - Phytossociological parameters and successional categories of tree species recorded in FLONA Pacotuba, municipality of Cachoeiro de Itapemirim, ES.

\begin{tabular}{|c|c|c|c|c|c|c|c|c|}
\hline Família & Nome científico & $\mathrm{CS}$ & $\mathrm{N}$ & $\mathrm{AB}$ & DR & FR & DoR & VI \\
\hline Euphorbiaceae & Senefeldera verticillata (Vell.) Croizat & St & 1062 & 9,04 & 29,75 & 1,77 & 14,65 & 46,16 \\
\hline Euphorbiaceae & Actinostemon klotzschii (Didr.) Pax & St & 703 & 4,07 & 19,69 & 1,77 & 6,59 & 28,05 \\
\hline Fabaceae & Anadenanthera peregrina (L.) Speg. & $\mathrm{Si}$ & 94 & 6,80 & 2,63 & 1,03 & 11,01 & 14,67 \\
\hline Anacardiaceae & Astronium concinnum Schott & St & 89 & 2,12 & 2,49 & 1,77 & 3,44 & 7,70 \\
\hline Rutaceae & Neoraputia alba (Nees \& Mart.) Emmerich ex Kallunki & St & 122 & 1,77 & 3,42 & 1,03 & 2,86 & 7,31 \\
\hline Fabaceae & Pseudopiptadenia contorta (DC.) G.P.Lewis \& M.P.Lima & $\mathrm{Si}$ & 39 & 2,57 & 1,09 & 1,47 & 4,17 & 6,74 \\
\hline Myristicaceae & Virola gardneri (A.DC.) Warb. & St & 93 & 1,48 & 2,61 & 1,33 & 2,39 & 6,32 \\
\hline Sapotaceae & Chrysophyllum lucentifolium Cronquist. & St & 97 & 0,68 & 2,72 & 1,77 & 1,10 & 5,58 \\
\hline Salicaceae & Casearia sp. & - & 67 & 1,24 & 1,88 & 1,18 & 2,01 & 5,06 \\
\hline Violaceae & Rinorea bahiensis (Moric.) Kuntze & St & 51 & 1,52 & 1,43 & 1,03 & 2,46 & 4,92 \\
\hline Malvaceae & Basiloxylon brasiliensis (All.) K.Schum. & St & 13 & 1,92 & 0,36 & 1,18 & 3,11 & 4,66 \\
\hline Nyctaginaceae & Ramisia brasiliensis Oliv. & $\mathrm{Si}$ & 44 & 0,40 & 1,23 & 1,47 & 0,66 & 3,36 \\
\hline Fabaceae & Myroxylon peruiferum L.f. & $\mathrm{Si}$ & 40 & 0,46 & 1,12 & 1,18 & 0,75 & 3,05 \\
\hline Phytolaccaceae & Gallesia integrifolia (Spreng.) Harms & St & 4 & 1,44 & 0,11 & 0,44 & 2,34 & 2,89 \\
\hline Anacardiaceae & Astronium graveolens Jacq. & $\mathrm{Si}$ & 29 & 0,39 & 0,81 & 1,33 & 0,63 & 2,76 \\
\hline Salicaceae & Macrothumia kuhlmannii (Sleumer) M.H.Alford & St & 28 & 0,49 & 0,78 & 1,18 & 0,79 & 2,75 \\
\hline Myrtaceae & Marlierea tomentosa Cambess. & St & 32 & 0,32 & 0,90 & 1,33 & 0,52 & 2,74 \\
\hline Meliaceae & Trichilia lepidota Mart. & St & 18 & 0,62 & 0,51 & 1,18 & 1,01 & 2,69 \\
\hline Fabaceae & Abarema limae Iganci \& M.P.Morim & - & 20 & 0,89 & 0,56 & 0,59 & 1,43 & 2,58 \\
\hline Sapotaceae & Pouteria filipes Eyma & St & 19 & 0,34 & 0,53 & 1,48 & 0,55 & 2,56 \\
\hline Nyctaginaceae & Andradea floribunda Allemão & $\mathrm{Si}$ & 5 & 1,10 & 0,14 & 0,59 & 1,79 & 2,52 \\
\hline Fabaceae & Copaifera lucens Dwyer & St & 21 & 0,45 & 0,59 & 1,18 & 0,72 & 2,49 \\
\hline Annonaceae & Oxandra nitida R.E.Fr. & - & 15 & 0,55 & 0,42 & 1,18 & 0,89 & 2,49 \\
\hline Combretaceae & Alseis sp. & St & 19 & 0,76 & 0,53 & 0,59 & 1,23 & 2,35 \\
\hline Sapotaceae & Sapotaceae sp. & - & 7 & 0,80 & 0,20 & 0,74 & 1,30 & 2,23 \\
\hline Moraceae & Sorocea guilleminiana Gaudich. & St & 31 & 0,20 & 0,87 & 1,03 & 0,32 & 2,22 \\
\hline Rhamnaceae & Ziziphus platyphylla Reissek & St & 15 & 0,33 & 0,42 & 1,18 & 0,53 & 2,13 \\
\hline Apocynaceae & Geissospermum laeve (Vell.) Miers & St & 16 & 0,48 & 0,45 & 0,88 & 0,77 & 2,10 \\
\hline Sapotaceae & Ecclinusa ramiflora Mart. & St & 22 & 0,13 & 0,62 & 1,18 & 0,22 & 2,01 \\
\hline Sapotaceae & Micropholis sp. & St & 16 & 0,41 & 0,45 & 0,88 & 0,66 & 2,00 \\
\hline Rutaceae & Zanthoxylum sp. & - & 12 & 0,45 & 0,34 & 0,88 & 0,73 & 1,95 \\
\hline Nyctaginaceae & Guapira noxia (Netto) Lundell & $\mathrm{Si}$ & 20 & 0,36 & 0,56 & 0,74 & 0,59 & 1,89 \\
\hline Bignoniaceae & Paratecoma peroba (Record) Kuhlm. & St & 8 & 0,70 & 0,22 & 0,44 & 1,13 & 1,79 \\
\hline Myristicaceae & Virola bicuhyba (Schott ex Spreng.) Warb. & St & 19 & 0,58 & 0,53 & 0,29 & 0,93 & 1,76 \\
\hline
\end{tabular}


Tabela 1 - Continuação...

Table 1 - Continued...

\begin{tabular}{|c|c|c|c|c|c|c|c|c|}
\hline Família & Nome científico & $\mathrm{CS}$ & $\mathrm{N}$ & $\mathrm{AB}$ & DR & FR & DoR & VI \\
\hline Sapotaceae & Micropholis gardneriana (A.DC.) Pierre & St & 15 & 0,16 & 0,42 & 1,03 & 0,26 & 1,71 \\
\hline Sapindaceae & Melicoccus oliviformis Kunth & - & 8 & 0,34 & 0,22 & 0,88 & 0,55 & 1,66 \\
\hline Bignoniaceae & Tabebuia cassinoides (Lam.) DC. & $\mathrm{Pi}$ & 11 & 0,27 & 0,31 & 0,88 & 0,44 & 1,63 \\
\hline Celastraceae & Maytenus cestrifolia Reissek & - & 14 & 0,11 & 0,39 & 1,03 & 0,18 & 1,60 \\
\hline Lecythidaceae & Couratari asterotricha Prance & $\mathrm{Si}$ & 13 & 0,10 & 0,36 & 1,03 & 0,17 & 1,56 \\
\hline Moraceae & Clarisia racemosa Ruiz \& Pav. & St & 14 & 0,06 & 0,39 & 1,03 & 0,10 & 1,52 \\
\hline Lecythidaceae & Cariniana legalis (Mart.) Kuntze & St & 4 & 0,56 & 0,11 & 0,44 & 0,91 & 1,47 \\
\hline Schoepfiaceae & Schoepfia brasiliensis A.DC. & St & 14 & 0,16 & 0,39 & 0,74 & 0,26 & 1,39 \\
\hline Euphorbiaceae & Actinostemon verticillatus (Klotzsch) Baill. & St & 7 & 0,10 & 0,20 & 1,03 & 0,15 & 1,38 \\
\hline Anacardiaceae & Spondias venulosa (Engl.) Engl. & $\mathrm{Si}$ & 2 & 0,71 & 0,06 & 0,15 & 1,15 & 1,35 \\
\hline Meliaceae & Trichilia pallens C.DC. & St & 15 & 0,08 & 0,42 & 0,74 & 0,12 & 1,28 \\
\hline Lauraceae & Ocotea complicata (Meisn.) Mez & St & 2 & 0,56 & 0,06 & 0,29 & 0,91 & 1,26 \\
\hline Lauraceae & Ocotea elegans $\mathrm{Mez}$ & St & 11 & 0,04 & 0,31 & 0,88 & 0,07 & 1,26 \\
\hline Fabaceae & Dalbergia nigra (Vell.) Allemão ex Benth. & St & 7 & 0,28 & 0,20 & 0,59 & 0,46 & 1,24 \\
\hline Fabaceae & Swartzia apetala Raddi & St & 7 & 0,08 & 0,20 & 0,88 & 0,12 & 1,20 \\
\hline Lecythidaceae & Lecythis lurida (Miers) S.A.Mori & St & 5 & 0,18 & 0,14 & 0,74 & 0,29 & 1,17 \\
\hline Meliaceae & Guarea guidonia (L.) Sleumer & $\mathrm{Si}$ & 12 & 0,14 & 0,34 & 0,59 & 0,22 & 1,15 \\
\hline Fabaceae & Parapiptadenia pterosperma (Benth.) Brenan & $\mathrm{Si}$ & 5 & 0,16 & 0,14 & 0,74 & 0,26 & 1,14 \\
\hline Euphorbiaceae & Cnidoscolus oligandrus (Müll.Arg.) Pax & - & 10 & 0,24 & 0,28 & 0,44 & 0,40 & 1,12 \\
\hline Lauraceae & Ocotea sp. & - & 12 & 0,11 & 0,34 & 0,59 & 0,18 & 1,11 \\
\hline Sapotaceae & Pouteria reticulata (Engl.) Eyma & St & 4 & 0,32 & 0,11 & 0,44 & 0,52 & 1,08 \\
\hline Sapotaceae & Chrysophyllum splendens Spreng. & St & 4 & 0,32 & 0,11 & 0,44 & 0,52 & 1,07 \\
\hline Fabaceae & Senegalia polyphylla (DC.) Britton \& Rose & $\mathrm{Si}$ & 4 & 0,21 & 0,11 & 0,59 & 0,33 & 1,04 \\
\hline Nyctaginaceae & Guapira opposita (Vell.) Reitz & $\mathrm{Si}$ & 10 & 0,09 & 0,28 & 0,59 & 0,15 & 1,02 \\
\hline Meliaceae & Trichilia silvatica C.DC. & St & 7 & 0,05 & 0,20 & 0,74 & 0,07 & 1,01 \\
\hline Myrtaceae & Myrtaceae sp. & - & 4 & 0,19 & 0,11 & 0,59 & 0,30 & 1,00 \\
\hline- & Outras (193 sp.) & - & 459 & 10,26 & 13,1 & 44,7 & 16,7 & 74,10 \\
\hline Total (2,4 ha) & & - & 3570 & 61,73 & 100 & 100 & 100 & 300 \\
\hline
\end{tabular}

Legenda: $\mathrm{N}=$ número de indivíduos, $\mathrm{AB}=$ área basal $\left(\mathrm{m}^{2}\right), \mathrm{DR}=$ densidade relativa, $\mathrm{FR}=$ frequiência relativa, $\mathrm{DoR}=$ dominância relativa, $\mathrm{VC}=$ valor de importância, $\mathrm{CS}=$ Categoria Sucessional,, $\mathrm{Pi}=$ Pioneira, $\mathrm{SI}=$ Secundária Inicial, $\mathrm{ST}=$ Secundária Tardia.

Legend: $N=$ number of individuals; $A B=$ basal area $\left(m^{2}\right), D R=$ relative density. $F R=$ relative frequency, DoR $=$ relative dominance, $V I=$ importance value, $C S=$ sucessional category, $P i=$ Pioneer, $S I=$ Initial Secondary, $S T=$ Late secondary, NC=Not characteristic of any of the ecological groups due to a lack of pertinent information.

apresentam maior densidade, sendo responsáveis por $70 \%$ do número total de indivíduos amostrados, sendo elas: Senefeldera verticillata (Vell.) Croizat, Actinostemon klotzschii (Didr.) Pax, Neoraputia alba (Nees \& Mart.) Emmerich ex Kallunki, Chrysophyllum lucentifolium
Cronquist., Anadenanthera peregrina (L.) Speg., Virola gardneri (A.DC.) Warb., Astronium concinnum Schott, Casearia sp., Rinorea bahiensis (Moric.) Kuntze, Ramisia brasiliensis Oliv., Myroxylon peruiferum L.f., Pseudopiptadenia contorta (DC.) G.P.Lewis \& M.P.Lima. 

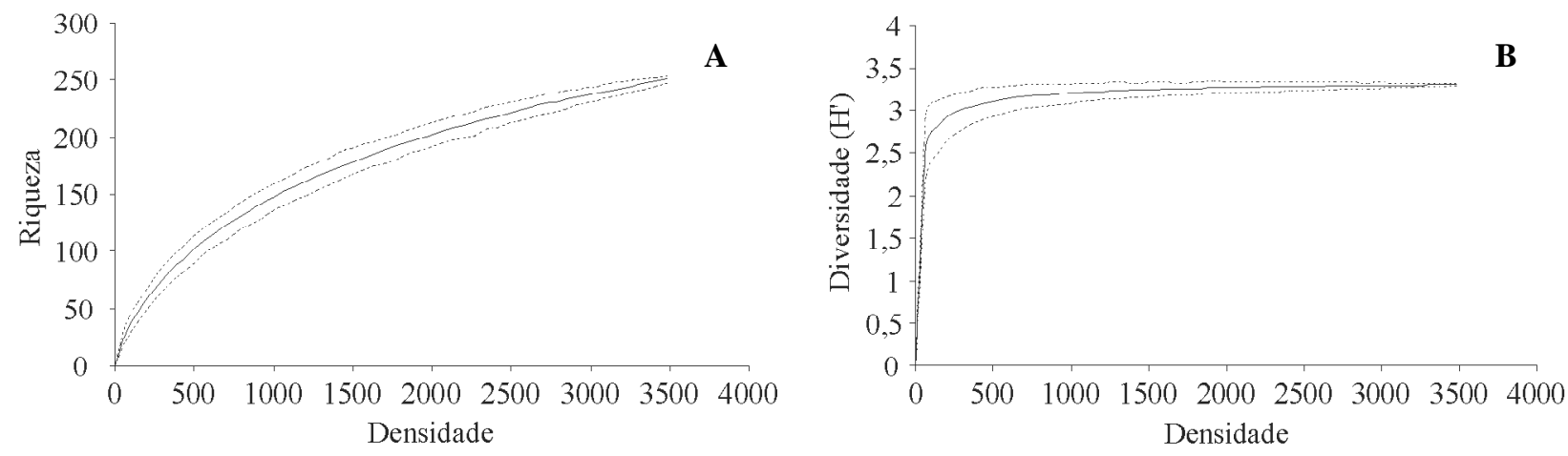

Figura 2 - Curvas de rarefação com valores medianos de riqueza (A) e diversidade (B) de espécies arbóreas e seus valores de limite de confiança $(95 \%)$ inferiores e superiores (- - -) no fragmento estudado.

Figure 2 - Rarefaction curves with median values of the tree species richness $(A)$ and diversity (B) and $95 \%$ upper and lower confidence values (- - ) for the studied fragment.

Tabela 2 - Parâmetros analisados em algumas Florestas Estacionais Semideciduais.

Table 2 - Analyzed parameters in some Semideciduous Seasonal Forest.

\begin{tabular}{llccccccl}
\hline Município-UF & \multicolumn{1}{c}{ Área Amostral } & DAP & AB & Ni & Nsp & H' & J & Referência \\
\hline Itatinga, SP & Fazenda Santa Irene (0,4 ha) & 4,8 & 31,93 & 2.271 & 97 & 3,77 & 0,82 & Ivanauskas et al. (1999) \\
Lavras-MG & PE Rio Bonito (2,4 ha) & 5 & 20,50 & 1.724 & 213 & 4,56 & 0,85 & Dalanesi et al. (2004) \\
Viçosa, MG & Sítio Bom Sucesso (0,5 ha) & 5 & 38,45 & 1.704 & 151 & 3,52 & 0,69 & Campos et al. (2006) \\
Tiradentes-MG & Serra de São José (0,9 ha) & 5 & 19,79 & 2.387 & 130 & 4,24 & 0,87 & Gonzaga et al. (2008) \\
Pirenópolis, GO & Fazenda Raio de Sol (0,4 ha) & 5 & 20,08 & 1.855 & 83 & 3,80 & 0,86 & Imaña-Encinas et al. (2008) \\
Uberaba-MG & Faz. Sucupira-Caçu (1,0 ha) & 4,8 & 45,80 & 805 & 90 & 3,33 & 0,73 & Dias Neto et al. 2009 \\
Búzios-RJ & APA do Pau Brasil (0,5 ha) & 5 & 23,60 & 2.386 & 98 & 3,60 & 0,79 & Kurtz et al. (2009) \\
S. José de Ubá-RJ & Bacia São Domingos (1,0 ha) & 5 & 27,30 & 1.144 & 198 & 3,60 & 0,87 & Dan et al. (2010) \\
C. de Itapemirim-ES & RPPN Cafundó (2,5 ha) & 5 & 33,02 & 1.823 & 258 & 4,13 & 0,74 & Archanjo et al. (2012) \\
C. de Itapemirim-ES & FLONA Pacotuba (2,4 ha) & 5 & 25,72 & 1.488 & 253 & 3,31 & 0,60 & Presente estudo \\
\hline
\end{tabular}

Legenda: $\mathrm{DAP}=$ diâmetro mínimo para inclusão na análise fitossociologica em $\mathrm{cm}, \mathrm{AB}=$ área basal total em $\mathrm{m}^{2}$ por ha, $\mathrm{Ni}=$ número de indivíduos por ha, $\mathrm{Nsp}=$ número de espécies, $\mathrm{H}^{\prime}$ = índice de diversidade de Shannon para espécies.

Legend: DAP = minimum diameter at breast height $(\mathrm{cm}), A B=$ total basal area in $m^{2}$ per hectare, $N i=$ number of individuals per hectare, $N s p=$ number of species, $H '=$ Shannon species diversity index, $J=$ uniformity of species .

Cabe ressaltar o destaque da espécie Senefeldera verticillata em densidade (30\% dos indivíduos), o que também contribuiu para redução da equabilidade $(0,60)$ encontrada nessa amostragem. Moreira et al. (2006) relataram uma perda da qualidade geral do fragmento da FLONA de Pacotuba, uma vez que a alta concentração de Euphorbiaceae normalmente ocorre em ambientes florestais impactados. $\mathrm{Na}$ RPPN Cafundó, esta espécie ocorreu entre as de maior VI, porém com menor destaque (4\% dos indivíduos) (ARCHANJO et al., 2012). Apesar de Senefeldera verticillata ser classificada como uma espécie secundária tardia, a mesma é considerada generalista, com predominância verificada em áreas perturbadas (GUEDES-BRUNI et al., 2006). Pessoa e Oliveira (2006) encontraram incidência acentuada de $S$. verticillata em fragmentos perturbados da Reserva Biológica de Poço das Antas - RJ, o que corrobora como os resultados obtidos neste estudo, em razão do histórico de perturbação da FLONA de Pacotuba.

Vale et al. (2009) esclarecem que as espécies que dominam uma comunidade arbórea podem apresentar estrutura horizontal diferenciada. Desse modo, observou-se na área em estudo que a espécie $S$. verticillata apresenta

\section{Cerne, Lavras, v. 19, n. 1, p. 157-168, jan./mar. 2013}


elevados valores de densidade, enquanto que $A$. peregrina ocorre com elevados valores de dominância. A espécie $N$. alba se destaca por apresentar valores elevados de densidade e dominância relativas. A segunda colocada em VI, A. klotzschii também ocorreu entre as de maior VI em uma FES em São Paulo (BERTANI et al., 2001) e em uma Floresta Ombrófila Aberta no Maranhão(GAMA, 2007). A espécie $A$. peregrina ocorreu com destaque em uma vegetação colonizadora de área degradada em Minas Gerais (ARAÚJO et al., 2006), bem como no Paraná (SILVA; SCARIOT, 2003) e, nesse levantamento, foi registrada preferencialmente em parcelas localizadas em áreas mais alteradas e topos de morro, porém com diâmetros expressivos, o que pode sugerir uma evolução no processo de sucessão inicial para o intermediário, visto que a ocorrência de muitos indivíduos jovens desta espécie surge logo após a perturbação.

Foi verificado um considerável número de espécies (89), ocorrendo com apenas um indivíduo na amostragem total (35,2\% da riqueza e $2,5 \%$ da densidade), consideradas localmente raras, segundo conceito de Martins (1993). Esses valores podem indicar que muitas populações podem vir a sofrer dificuldades de conservação em longo prazo (CARVALHO et al., 2007; SOUZA et al., 2003). As espécies raras estão mais suscetíveis à extinção local, especialmente em decorrência dos efeitos da fragmentação (SCARIOT et al., 2005), visto que o número reduzido de indivíduos, as constantes perturbações e o isolamento genético podem comprometer a conservação biológica destas (MACHADO et al., 2004). Segundo Giulietti et al. (2009), espécies mais suscetíveis a distúrbios antrópicos podem ser consideradas raras, devendo ser tratadas como vulneráveis. Ressaltase ainda, a ocorrência de espécies consideradas ameaçadas de extinção pelo Ministério do Meio Ambiente (Instrução Normativa $n^{\circ} 6$, de 23 de setembro de 2008) dentre as espécies raras e de baixa densidade (menos de 5 indivíduos) como: Melanoxylon brauna Schott, Moldenhawera papillanthera L.P.Queiroz, GP.Lewis \& Allkin, Myrcia follii G.Barroso \& Peixoto, Trigoniodendron spiritusanctense E.F.Guim. \& J.Miguel. Além de espécies madeiráveis, com alto valor comercial: Aspidosperma illustre (Vell.) Kuhlm. \& Pirajá, Aspidosperma polyneuron Müll.Arg., Cabralea canjerana (Vell.) Mart., Cariniana legalis (Mart.) Kuntze e Dalbergia nigra (Vell.) Allemão ex Benth (BRASIL, 2008).

As espécies $M$. brauna e D. nigra também foram destacadas por Carvalho et al. (2007) por terem sido raramente registradas em levantamentos florísticos no estado do Rio de Janeiro, tendendo ao declínio em decorrência dos efeitos da fragmentação. Sendo assim, verifica-se a necessidade de mecanismos de apoio a conservação dessas espécies, como a implantação dos corredores ecológicos previstos para a região (ARCHANJO et al., 2012), bem como plantios de enriquecimento.

Por meio da elaboração do histograma representando a distribuição diamétrica, foram obtidas 12 classes e $10 \mathrm{~cm}$ de intervalo entre as mesmas. A curva de distribuição dos indivíduos considerando toda a comunidade (Figura 3) seguiu o padrão característico de florestas tropicais inequiâneas, ou seja, apresentou distribuição exponencial na forma de $\mathbf{J}$ invertido (ASSMANN, 1970; SCOLFORO, 1998), em que a maior frequência de indivíduos ocorreu nas menores classes de diâmetro. Amenor classe diamétrica mostrada no histograma de distribuição apresentou a maior frequência de indivíduos, o que indica que a maioria das populações pode estar em fase inicial de estabelecimento. A maior concentração de indivíduos nas primeiras classes de diâmetro pode caracterizar uma comunidade-estoque, o que é padrão em florestas tropicais estáveis com idade e composição de espécies variadas (SOUZA et al., 2012), destacando, nesse caso, a capacidade autorregenerativa da área estudada.

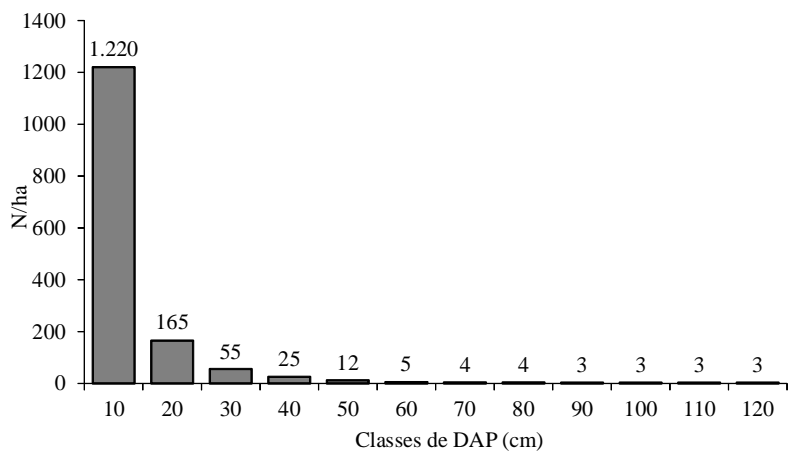

Figura 3 - Densidade absoluta de indivíduos por hectare (DA) por classe de diâmetro para o fragmento da Floresta Nacional de Pacotuba.

Figure 3 - Density of individuals per hectare $(D A)$ in diameter classes for the Pacotuba National Forest fragment.

Os valores do quociente "q" de Liocourt variaram entre as classes diamétricas da comunidade (Tabela 3 ), indicando tendências diferenciadas (CARVALHO; NASCIMENTO, 2009). O valor de "q1" $(0,13)$ mostrou bem abaixo do valor médio de "q" calculado $(0,61)$, indicando uma elevada taxa de recrutamento da comunidade, sendo um reflexo da grande concentração de indivíduos na primeira classe de diâmetro. Característica

Cerne, Lavras, v. 19, n. 1, p. 157-168, jan./mar. 2013 
Tabela 3 - Valores do quociente "q" de Liocourt da comunidade, quanto à distribuição diamétrica, para o fragmento da Floresta Nacional de Pacotuba.

Table 3 - Liocourt's " $q$ " quotient values for the studied community, in relation to the diametric distribution, for the Pacotuba National Forest fragment.

\begin{tabular}{lccccccccccccc}
\hline "q" de Liocourt & $\mathrm{q} 1$ & $\mathrm{q} 2$ & $\mathrm{q} 3$ & $\mathrm{q} 4$ & $\mathrm{q} 5$ & $\mathrm{q} 6$ & $\mathrm{q} 7$ & $\mathrm{q} 8$ & $\mathrm{q} 9$ & $\mathrm{q} 10$ & $\mathrm{q} 11$ & q12 & qmédia \\
\hline Valores & 0,14 & 0,33 & 0,46 & 0,47 & 0,42 & 0,80 & 0,93 & 0,81 & 1,00 & 1,00 & 1,00 & 00 & 0,61 \\
\hline
\end{tabular}

semelhante foi encontrada por Carvalho e Nascimento (2009), estudando um remanescente de Floresta Atlântica Submontana em Silva Jardim, RJ. Algo conforme, embora em menor intensidade, ocorreu para os valores de "q2" $(0,33)$. Já, os valores de "q3" a "q4" mostraram maior aproximação do valor médio, enquanto os valores " $q 6$ " a "q11" foram superiores ao valor médio, indicando baixa taxa de recrutamento nas classes finais, pela menor concentração de indivíduos nas classes de maior diâmetro.

Apesar da distribuição diamétrica apresentar a forma de $\mathbf{J}$ invertido, ela não se mostrou balanceada, ou seja, o fator de redução do número de indivíduos de uma classe de diâmetro para a seguinte não se mostrou constante (MARANGON et al., 2008; SCOLFORO et al., 1997). Observa-se ainda, um número muito reduzido de indivíduos nas classes de maior diâmetro, indicando a ocorrência de perturbações antrópicas na área de estudo.

Com relação às categorias sucessionais, observouse que a maioria das espécies $(39,5 \%)$ e dos indivíduos $(79,1 \%)$ foi classificada como tardia. A baixa densidade de espécies pioneiras $(2,8 \%$ das espécies e $0,6 \%$ dos indivíduos) e secundárias iniciais (19\% das espécies e $11 \%$ dos indivíduos) pode indicar condições menos perturbadas das florestas tropicais (HARTSHORN, 1980; HUBBEL et al., 1999). Entretanto, na área estudada, a menor representatividade dos grupos iniciais de sucessão está relacionada à elevada dominância de $S$. verticillata, uma espécie considerada generalista, refletindo o histórico de perturbação desse fragmento no passado, que pode ter favorecido a colonização por essa espécie.

Acredita-se que a estrutura fitossociológica do fragmento analisado pode ser, em parte, explicada pelo histórico de perturbações locais a que foi submetido. A fragmentação da área, a exploração de seus recursos naturais e sua consolidação como unidade de conservação ter ocorrido recentemente, provavelmente favoreceram a ocorrência de impactos antrópicos.

\section{CONCLUSÕES}

A diversidade e a heterogeneidade florísticas relativamente baixas indicam a dominância ecológica de poucas espécies na comunidade, principalmente a generalista $S$. verticillata, refletindo o histórico de perturbação do fragmento. Apesar de apresentar características de áreas perturbadas, a maior concentração de indivíduos nas classes iniciais de diâmetro indica um balanço positivo entre recrutamento e mortalidade, destacando que a área estudada possui potencial regenerativo. Verifica-se a necessidade dessa área ser priorizada em programas conservacionistas na região e a urgência na adoção de medidas de manejo criteriosas para a restauração desse remanescente.

\section{AGRADECIMENTOS}

Aos especialistas do Instituto de Pesquisas do Jardim Botânico-RJ pelo auxílio na identificação botânica, a Fabio Silveira Castro pela elaboração do mapa, a Adriano Ribeiro de Mendonça pelo auxílio nas análises diamétricas, a Fundação de Apoio à Ciência e Tecnologia do Espírito Santo (FAPES) e ao Departamento de Engenharia Florestal da Universidade Federal do Espírito Santo (UFES) pelo apoio financeiro e logístico e aos avaliadores anônimos do periódico, pela revisão e contribuições ao texto.

\section{REFERÊNCIAS}

ANGIOSPERM PHYLOGENY GROUP. An update of the Angiosperm Phylogeny Group classification for the orders and families of flowering plants: APG III. Journal of the

Linnean Society, London, v. 161, n. 2, p. 105-121, 2009.

ARAÚJO, F. S.; MARTINS, S. V.; MEIRA NETO, J. A. A.; LANI, J. L.; PIRES, I. E. Estrutura da vegetação arbustivoarbórea colonizadora de uma área degradada por mineração de caulim, Brás Pires, MG. Revista Árvore, Viçosa, v. 30, n. 1, p. 107-116, 2006.

ARCHANJO, K. M. P. A.; SILVA, G. F.; CHICHORRO, J. F.; SOARES, C. P. B. Estrutura do componente arbóreo da Reserva Particular do Patrimônio Natural Cafundó, Cachoeiro de Itapemirim, Espírito Santo, Brasil. Floresta, Curitiba, v. 42, n. 1, p. 145-160, 2012.

Cerne, Lavras, v. 19, n. 1, p. 157-168, jan./mar. 2013 
ASSMANN, E. The principles of forest yield: studies in the organic production, structure, increment and yield of forest stands. Braunschweig: Pergamon, 1970. 506 p.

BERTANI, D. F.; RODRIGUES, R. R.; BATISTA, J. L. F; SHEPHERD, G. J. Análise temporal da heterogeneidade florística e estrutural em uma floresta ribeirinha. Revista Brasileira de Botânica, São Paulo, v. 24, p. 11-23, 2001.

BRASIL. Ministério do Meio Ambiente. Instrução Normativa $\mathbf{N}^{\mathbf{0}}$ 6, de 23 de setembro de 2008. Brasília, 2008. Disponível em: <http://www.iap.pr.gov.br/arquivos/File/ Legislacao_ambiental/Legislacao_federal/instrucao_normativa/ instrucao_normativa_06_de_23_de_setembro_de_2008.pdf >. Acesso em: 10 out. 2011.

CAMPOS, E. P.; SILVA, A. F; MEIRA NETO, J. A. A.; MARTINS, S. V. Florística e estrutura horizontal de vegetação arbórea de uma ravine em um fragmento florestal no município de Viçosa, MG. Revista Árvore, Viçosa, v. 30, n. 6, p. 1045 1054, 2006.

CARVALHO, F. A.; BRAGA, J. M. A.; GOMES, J. M. L.; SOUZA, J. S.; NASCIMENTO, M. T. N. Comunidade arbórea de uma floresta de baixada aluvial no município de Campos dos Goytacazes, RJ. Cerne, Larvas, v. 12, n. 2, p. 157-166, 2006.

CARVALHO, F. A.; BRAGA, J. M. A.; NASCIMENTO, M. T. Estrutura da Comunidade Arbórea de Fragmentos de Floresta Atlântica Ombrófila Submontana na Região de Imbaú. Rodriguésia, Rio de Janeiro, v. 57, n. 3, p. 503-518, 2009.

\section{CARVALHO, F. A.; NASCIMENTO, M. T. Estrutura} diamétrica da comunidade e das principais populações arbóreas de um remanescente de floresta atlântica submontana, Silva Jardim-RJ, Brasil. Revista Árvore, Viçosa, v. 33, n. 2, p. $327-$ $337,2009$.

CARVALHO, F. A.; NASCIMENTO, M. T.; BRAGA, J. M. A. Estrutura e composição florística do estrato arbóreo de um remanescente de Mata Atlântica Submontana no município de Rio Bonito, RJ, Brasil, Mata Rio Vermelho. Revista Árvore, Viçosa, v. 31, n. 4, p. 717-730, 2007.

CIENTEC. Software Mata Nativa 2.06: sistema para análise fitossociológica, elaboração de inventários e planos de manejo de florestas nativas. Viçosa, MG, 2006. 295 p.
CONSELHO NACIONAL DO MEIO AMBIENTE.

Resolução No 29, de 07 de Dezembro de 1994. Brasília, 1994.

CORDEIRO, N. J.; HOWE, H. F. Low recruitment of trees dispersed by animals on African forest fragments.

Conservation Biology, Cambridge, v. 15, n. 6, p. 1733-1741, 2001.

DALANESI, P. E.; OLIVEIRA FILHO, A. T.; FONTES, M. A. L. Flora e estrutura do componente arbóreo da floresta do Parque Ecológico Quedas do Rio Bonito, Lavras, MG e correlações entre a distribuição das espécies e variáveis ambientais. Acta Botanica Brasilica, Porto Alegre, v. 18, p. 737-757, 2004.

DAN, M. L.; BRAGA, J. M. A.; NASCIMENTO, M. T. Estrutura da comunidade arbórea de fragmentos de floresta estacional semidecidual na bacia hidrográfica do Rio São Domingos, São José de Ubá, Rio de Janeiro, Brasil.

Rodriguésia, Rio de Janeiro, v. 61, n. 4, p. 749-766, 2010.

DIAS NETO, O. C.; SCHIAVINI, I.; LOPES, S. F.; VALE, V. S.; GUSSON, A. E.; OLIVEIRA, A. P. Estrutura fitossociológica e grupos ecológicos em fragmento de floresta estacional semidecidual, Uberaba, Minas Gerais, Brasil. Rodriguésia, Rio de Janeiro, v. 60, p. 1087-1100, 2009.

\section{EMPRESA BRASILEIRA DE PESQUISA}

AGROPECUÁRIA. Sistema brasileiro de classificação de solos. Rio de Janeiro, 2006. 306 p.

FAHRIG, L. Effects of habitat fragmentation on biodiversity. Annual Review of Ecology, Evolution and Systematic, Palo Alto, v. 34, p. 487-515, 2003.

FELFILI, J. M.; REZENDE, R. P. Conceitos e métodos em fitossociologia. Brasília: UnB, 2003. 64 p.

FUNDAÇÃO SOS MATA ATLÂNTICA; INSTITUTO NACIONAL DE PESQUISAS ESPACIAIS. Atlas dos remanescentes florestais da Mata Atlântica período 20052008. São Paulo, 2009. 156 p.

GAMA, J. R. V. Fitossociologia de duas fitocenoses de Floresta Ombrófila Aberta no município de Codó, Estado do Maranhão. Revista Árvore, Viçosa, v. 31, n. 3, p. 465-477, 2007.

Cerne, Lavras, v. 19, n. 1, p. 157-168, jan./mar. 2013 
GANDOLFI, S.; LEITÃO FILHO, H. F.; BEZERRA, C. L. F. Levantamento florístico e caráter sucessional das espécies arbustivo-arbóreas de uma Floresta Semidecídua no município de Guarulhos, SP. Revista Brasileira de Biologia, Rio de Janeiro, v. 55, p. 753-767, 1995.

GIULIETTI, A. M.; RAPINI, A.; ANDRADE, M. J. G.; QUEIROZ, L. P.; SILVA, J. M. C. (Org.). Plantas raras do Brasil. Belo Horizonte: Universidade Estadual de Feira de Santana, 2009. 496 p.

GONZAGA, A. P. D.; OLIVEIRA-FILHO, A. T.; MACHADO, E. L. M.; HARGREAVES, P.; MACHADO, J. N. M. Diagnóstico florístico-estrutural do componente arbóreo da floresta da Serra de São José, Tiradentes, MG, Brasil. Acta Botanica Brasilica, Porto Alegre, v. 22, n. 2, p. 505-520, 2008.

GOTELLI, N. J.; ENTSMINGER, G. L. EcoSim: null models software for ecology. Version 7.0. Kesey-Bear: Acquired Intelligence, 2011.

GUEDES-BRUNI, R. R.; SILVA NETO, S. J.; MORIM, M. P.; MANTOVANI, W. Composição florística e estrutura de dossel em trecho de Floresta Ombrófila Densa Atlântica sobre morrote mamelonar na Reserva Biológica de Poço das Antas, Silva Jardim, Rio de Janeiro, Brasil. Rodriguésia, Rio de Janeiro, v. 57, n. 3, p. 429-442, 2006.

HARTSHORN, G. S. Neotropical forest dynamics. Biotropica, Washington, n. 12, p. 30-32, 1980. Supplement.

HUBBEL, S. P. et al. Light gaps disturbance, recruitment limitations and tree diversity in a neotropical forest. Science, New York, v. 283, p. 554-557, 1999.

IMAÑA-ENCINAS, J.; SANTANA, O. A.; MACEDO, L. A.; PAULA, J. E. Distribuição diamétrica de um trecho da Floresta Estacional Semidecidual na área do Ecomuseu do Cerrado. Cerne, Lavras, v. 14, n. 1, p. 33-45, 2008.

INSTITUTO CAPIXABA DE PESQUISA, ASSISTÊNCIA TÉCNICA E EXTENSÃO RURAL. Caracterização climática do município de Cachoeiro de Itapemirim. Disponível em: <http://hidrometeorologia.incaper.es.gov.br/ caracterizacao/cacho_itap_carac.php>. Acesso em: 20 fev. 2012.

INSTITUTO DE PESQUISAS DA MATA ATLÂNTICA. Conservação da Mata Atlântica no Estado do Espírito

Cerne, Lavras, v. 19, n. 1, p. 157-168, jan./mar. 2013
Santo: cobertura florestal e unidades de conservação. Vitória, 2005. $152 \mathrm{p}$.

IVANAUSKAS, N. M.; RODRIGUES, R. R.; NAVE, A. G. Fitossociologia de um trecho de floresta Estacional Semidecidual em Itatinga, São Paulo, Brasil. Scientia Florestalis, Piracicaba, v. 56, p. 83-99, 1999.

KENT, M.; COKER, P. Vegetation description and analysis. London: Belhaven Press, 1992. 363 p.

KÖPPEN, W. Climatologia: con un estudio de los climas de la tierra. Ciudad del México: Fondo de Cultura Econômica, 1948. 479 p.

KURTZ, B. C.; SÁ, C. F. C.; SILVA, D. O. Fitossociologia do componente arbustivo-arbóreo de florestas semidecíduas costeiras da Região de Emerenças, área de proteção ambiental do Pau Brasil, Armação dos Búzios, Rio de Janeiro, Brasil. Rodriguésia, Rio de Janeiro, v. 60, n. 1, p. 129-146, 2009.

LAURANCE, W. F.; LOVEJOY, L. E.; VASCONCELOS, H. L.; BRUNA, E. M.; DIDHAM, R. K.; STOUFFER, P. C.; GASCON, C.; BIERREGAARD JUNIOR, R. O.; LAURANCE, S. G.; SAMPAIO, E. Ecosystem decay of Amazonian forest fragments: a 22-year investigation. Conservation Biology, Cambridge, v. 16, n. 3, p. 605-618, 2002.

LAURANCE, W. F.; NASCIMENTO, H. E. M.; LAURANCE, S. G.; ANDRADE, A. C.; FEARNSIDE, P. M. Rain forest fragmentation and the proliferation of successional trees. Ecology, London, v. 87, n. 2, p. 469-482, 2006.

LAURANCE, W. F.; YENSEN, E. Predicting the impacts of edges in fragmented habitats. Biological Conservation, Boston, v. 55, n. 1, p. 77-92, 1991.

LIBANO, A. M.; FELFILI, J. M. Mudanças temporais na composição florística e na diversidade de um cerrado sensu stricto do Brasil Central em um período de 18 anos: 19852003. Acta Botanica Brasílica, Porto Alegre, v. 20, n. 4, p. 927-936, 2006.

MACHADO, E. L. M.; OLIVEIRA FILHO, A. T.; CARVALHO, W. A. C.; SOUZA, J. S.; BORÉM, R. A. T.; BOTEZELLI, L. Análise comparativa da estrutura e flora do compartimento arbóreo-arbustivo de um remanescente florestal na Fazenda Beira Lago, Lavras, MG. Revista Árvore, Viçosa, v. 28, p. 499-516, 2004. 
MARANGON, L. C.; FELICIANO, A. L. P.; BRANDÃO, C. F. L. S.; ALVES JÚNIOR, F. T. relações florísticas, estrutura diamétrica e hipsométrica de um fragmento de floresta estacional semidecidual em Viçosa, MG. Floresta, Curitiba, v. 38, n. 4, p. 699-709, 2008.

MARANGON, L. C.; SOARES, J. J.; FELICIANO, A. L. P.; BRANDÃO, C. F. L. S. Estrutura fitossociológica e classificação sucessional do componente arbóreo de um fragmento de Floresta Estacional Semidecidual, no município de Viçosa, Minas Gerais. Cerne, Lavras, v. 13, n. 2, p. 208$221,2007$.

MARTINS, F. R. Estrutura de uma floresta mesófila. Campinas: UNICAMP, 1993. 245 p.

MELO, F. P. L.; DIRZO, R.; TABARELLI, M. Biased seed rain in forest edges: evidence from the Brazilian Atlantic forest.

Biological Conservation, Boston, v. 132, n. 1, p. 50-60, 2006.

METZGER, J. P.; MARTENSEN, A. C.; DIXO, M.; BERNACCI, L. C.; RIBEIRO, M. C.; TEIXEIRA, A. M. G.; PARDINI, R.Time-lag in biological responses to landscape changes in a highly dynamic Atlantic forest region. Biological Conservation, Boston, v. 142, p. 1166-1177, 2009.

MOREIRA, L. N.; MORENO, M. R.; REDLING, J. S. H. Estrutura populacional de Senefeldera multiflora em um trecho de borda na Mata Atlântica Estacional Semidecidual da Floresta Nacional de Pacotuba, Cachoeiro de Itapemirim, Espírito Santo. In: CONGRESSO NACIONAL DE BOTÂNICA, 57., 2006, Gramado. Anais... Gramado: SNB, 2006. 1 CD-ROM.

MORELLATO, L. P. C.; HADDAD, C. F. B. Introduction: the Brazilian Atlantic Forest. Biotropica, Washington, v. 32, p. 786-792, 2000.

MUELLER-DOMBOIS, D.; ELLEMBERG, H. Aims and methods of vegetation ecology. New York: J. Wiley, 1974. $547 \mathrm{p}$.

MURCIA, C. Edge effects in fragmented forests: implications for conservation. Trends in Ecology and Evolution, London, v. 10 , p. 58-62, 1995.

NASCIMENTO, M. C.; SOARES, V. P.; RIBEIRO, C. A. A. S.; SILVA, E. Mapeamento dos fragmentos de vegetação florestal nativa da bacia hidrográfica do Rio Alegre, Espírito Santo, a partir de imagens do satélite IKONOS II. Revista Árvore, Viçosa, v. 30, n. 3, p. 389-398, 2006.

NASCIMENTO, M. T.; LIMA, H. C. Floristic and structural relationships of a tabuleiro forest in northeastern Rio de Janeiro, Brazil. In: THOMAS, W. W. (Ed.). The Atlantic coastal forest of northeastern Brazil. New York: New York Botanical Garden, 2008. p. 395-416.

OLIVEIRA-FILHO, A. T.; FONTES, M. A. L. Patterns of floristic differentiation among Atlantic forests in southeastern Brazil, and the influence of climate. Biotropica, Washington, v. 32, n. 4b, p. 793-810, 2000.

PESSOA, S. V. A.; OLIVEIRA, R. R. Análise estrutural da vegetação arbórea em três fragmentos florestais na Reserva Biológica de Poço das Antas, Rio de Janeiro, Brasil.

Rodriguésia, Rio de Janeiro, v. 57, n. 3, p. 391-411, 2006.

PEZZOPANE, J. E. M.; SANTOS, E. A.; ELEUTÉRIO, M. M.; REIS, E. F.; SANTOS, A. R. Espacialização da temperatura do ar no Estado do Espírito Santo. Revista Brasileira de Agrometeorologia, Sete Lagoas, v. 12, n. 1, p. 151-158, 2004.

PINTO, S. I. C.; MARTINS, S. V.; SILVA, A. G.; BARROS, N. F; DIAS, H. C. T.; SCOSS, L. M. Estrutura do componente arbustivo-arbóreo de dois estádios sucessionais de floresta estacional semidecidual na Reserva Florestal Mata do Paraíso, Viçosa, MG, Brasil. Revista Árvore, Viçosa, v. 31, n. 5, p. 823-833, 2007.

PROJETO CORREDORES ECOLÓGICOS. Síntese dos encontros regionais realizados com os municípios do Estado do Espírito Santo. Cariacica, 2005. 52 p.

RIBEIRO, M. C.; METZGER, J. P.; MARTENSEN, A. C.; PONZONI, F. J.; HIROTA, M. M. The Brazilian Atlantic Forest: how much is left, and how is the remaining forest distributed?: implications for conservation. Biological Conservation, Boston, v. 142, n. 6, p. 1141-1153, 2009.

ROLIM, S. G.; IVANAUSKAS, N. M.; RODRIGUES, R. R.; NASCIMENTO, M. T.; GOMES, J. M. L.; FOLLI, D. A.; COUTO, H. T. Z. Composição florística do estrato arbóreo da Floresta Estacional Semidecidual na Planície Aluvial do rio Doce, Linhares, ES, Brasil. Acta Botanica Brasilica, Porto Alegre, v. 20, n. 3, p. 549-561, 2006.

Cerne, Lavras, v. 19, n. 1, p. 157-168, jan./mar. 2013 
SCARIOT, A.; FREITAS, S. R.; MARIANO NETO, E.; NASCIMENTO, M. T.; OLIVEIRA, L. C.; SANAIOTTI, T.; SEVILHA, A. C.; VILLELA, D. M. Vegetação e flora. In: RAMBALDI, D. M.; OLIVEIRA, D. A. S. de (Org.).

Fragmentação de ecossistemas: causas, efeitos sobre a biodiversidade e recomendações de políticas públicas. Brasília: MMA/SBF, 2005. p. 103-123.

SCHETTINO, L. F; GONÇALVES, F. C. Florestas e reflorestamento: informações básicas ao produtor rural. Vitória: UFES, 2002. 172 p.

SCOLFORO, J. R. S. Manejo florestal. Lavras: UFLA/ FAEPE, 1998. 438 p.

SCOLFORO, J. R.; OLIVEIRA, A. D. S.; SILVA, S. T. O manejo da vegetação nativa através de corte seletivo. Colombo: EMBRAPA-CNPF, 1997. 253 p.

\section{SILVA, A. F; FONTES, N. R.; LEITÃO-FILHO, H.}

Composição florística e estrutura horizontal do estrato arbóreo de um trecho da Mata da Biologia da Universidade Federal de Viçosa, zona da mata de Minas Gerais. Revista Árvore, Viçosa, v. 24, n. 4, p. 397-405, 2000.

SILVA, L. A.; SCARIOT, A. Composição florística e estrutura da comunidade arbórea em uma floresta estacional decidual em afloramento calcário: fazenda São José, São Domingos, GO, Bacia do Rio Paranã. Acta Botanica Brasilica, Porto Alegre, v. 17, n. 2, p. 305-313, 2003.

SOUZA, J. S.; ESPÍRITO-SANTO, J. D. B.; FONTES, M. A. L.; OLIVEIRA-FILHO, A. T.; BOTEZELLI, L. Análise das variações florísticas e estruturais da comunidade arbórea de um fragmento de Floresta Semidecídua às margens do Rio Capivari, Lavras, MG. Revista Árvore, Viçosa, v. 27, n. 2, p. 185-206, 2003.

SOUZA, P. B.; SOUZA, A. L.; MEIRA NETO, J. A. A. Estrutura diamétrica dos estratos e grupos ecológicos de uma área de floresta estacional semidecidual, em Dionísio, MG. Revista Árvore, Viçosa, v. 36, n. 1, p. 151-160, 2012.

VALE, V. S.; SCHIAVINI, I.; LOPES, S. F.; DIAS NETO, O. C.; OLIVEIRA, A. P.; GUSSON, A. E. Composição florística e estrutura do componente arbóreo em um remanescente primário de Floresta Estacional Semidecidual em Araguari, Minas Gerais, Brasil. Hoehnea, São Paulo, v. 36, n. 3, p. $417-$ 429, 2009.

VELOSO, H. P.; RANGEL FILHO, A. L. R.; LIMA, J. C. A. Classificação da vegetação brasileira, adaptada a um sistema universal. Rio de Janeiro: IBGE, 1991. 123 p.

WHITMORE, T. C. Tropical forest disturbance, disappearance, and species loss. In: LAURANCE, W. F.; BIERREGAARD, R. O. (Ed.). Tropical forest remnants: ecology, management and conservation of fragmented communities. Chicago: University of Chicago, 1997. p. 312.

WHITMORE, T. C.; BURSLEM, D. F. R. P. Major disturbances in tropical rainforests. In: NEWBERY, D. M.; PRINS, H. H. T.; BROWN, N. (Ed.). Dynamics of tropical communities. Oxford: Blackwell, 1998. p. 549-565. 